\title{
DESIGN OF C-SLOTTED MICROSTRIP ANTENNA USING ARTIFICIAL NEURAL NETWORK MODEL
}

\author{
Pritam Singha Roy ${ }^{1}$, Samik Chakraborty ${ }^{2}$ \\ ${ }^{1}$ Assistant Professor, Electronics Engineering, DIET, West Bengal, India \\ e-mail:prittam.pritam@gmail.com \\ ${ }^{2}$ Professor, Communication Engineering, Indian Maritime University, West Bengal, India \\ e-mail:chakra_samik@yahoo.com
}

\begin{abstract}
In this paper, neural network model has been used to estimation of resonance frequency of a coaxial feed $C$-slotted Microstrip Antenna. The Multi-Layer Perceptron Feed forward back Propagation (MLPFFBP) and Radial basis function Artificial Neural Network (RBFANN) have been used to implement the neural network model. A relative performance analysis of the proposed neural network for different training algorithms. Number of neurons and number of hidden layer is also carried out for estimating the resonance frequency. The method of moment (MOM) based IE3D software was used to generate data dictionary for training and validation set of ANN. The results obtain using ANN are compared with simulation feeding and found quite satisfactory and also it is concluded that RBFANN network is more accurate and fast compared to MLPFFBP network algorithm.
\end{abstract}

Index Terms: Artificial Neural Network, C slot, Microstrip Antenna, Multilayer Feed Forward Networks, Radial basis function Artificial Neural Network, Resonance frequency.

\section{INTRODUCTION}

Microstrip antennas due to their many attractive features have drawn attention of industries for an ultimate solution for wireless communication. The existing era of wireless communication has led to the design of an efficient, wide band, low cost and small volume antennas which can readily be incorporated into a broad spectrum of systems [1, 2].sufficient amount of work [3-10] indicates how ANN have been used efficiently to design rectangular Microstrip antenna for the determination of different patch dimensions i,e length, width,resonant frequency, radiation efficiency etc.

In this paper, an attempt has been made to exploit the capability of artificial neural networks to calculate the resonating frequency of coaxial feed C-slotted Microstrip patch antenna. The trained ANN is used to determine different important antenna characteristics for various structural input variables.Neoro models are computationally much more efficient than EM models once they are trained with reliable learning data obtained from a "fine" model by either EM simulation or measurement $[3,4,5,6]$.The neuro models can be used for efficient and accurate optimization and design within the range of training.

In this work, the authors extend the work on the use of the artificial neural network (ANN) technique taking into account different variants of back propagation training algorithm with MLPFFBP and RBF ANN model are stressed upon in place of conventional numerical techniques for the C-slot microstrip antenna design. Then, with the ANN results, simulated results from the IE3D software are compared.

\section{DESIGN AND DATA GENERATION}

Designing of micro strip patch antenna depends on three parameters. In this paper The rectangular patch Microstrip antenna is designed to resonate at $6.8 \mathrm{GHz}$ frequency with dielectric constant 2.2,Substrate thickness $\mathrm{h}=1.5 \mathrm{~mm}$ ,L=16.01 $\mathrm{mm}$ and $\mathrm{W}=19.73 \mathrm{~mm}$. The width (W) and length (L) of antenna are calculated from conventional equations [11].

$$
\begin{aligned}
& w=\frac{c}{2 f_{o} \sqrt{\frac{\left(\varepsilon_{r}+1\right)}{2}}} \\
& \varepsilon_{\text {reff }}=\frac{\varepsilon_{p}+1}{2}+\frac{\varepsilon_{p}-1}{2}\left[1+12 \frac{h}{W}\right]^{-\frac{1}{2}} \\
& L_{\text {eff }}=\frac{c}{2 f_{o} \sqrt{\varepsilon_{\text {reff }}}} \\
& (\Delta L)=0.412 h \frac{\left(\varepsilon_{\text {reff }}+0.3\right)\left(\frac{W}{h}+0.264\right)}{\left(\varepsilon_{\text {reff }}-0.256\right)\left(\frac{W}{h}+0.5\right)} \\
& L=L_{\text {eff }}-2 \Delta L
\end{aligned}
$$

For generating data, we simulated the frequency domain response of the antenna for various patch dimensions, using method of moments based simulation software IE3D ${ }^{\mathrm{TM}}$. For training and testing of the ANN, 100 data sets are generated 
by simulation using IE3D ${ }^{\mathrm{TM}}$ simulation software. Four Antenna dimension as $\mathrm{L} 1$ is the horizontal slot length, $\mathrm{W}_{1}$ is

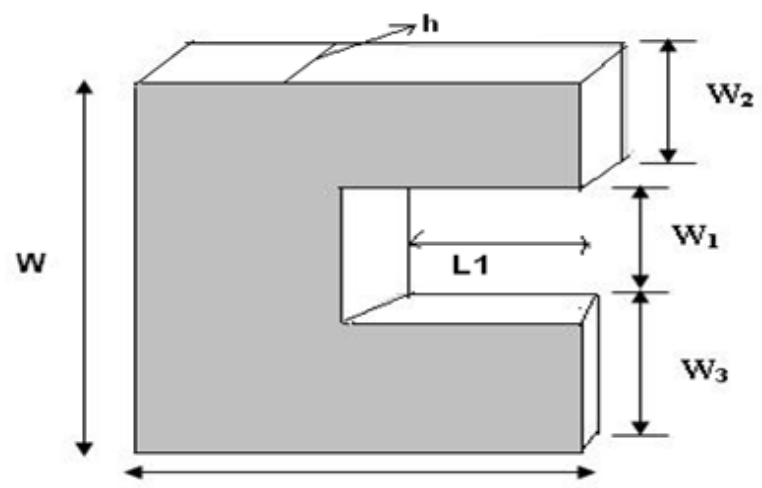

$\mathbf{L}$

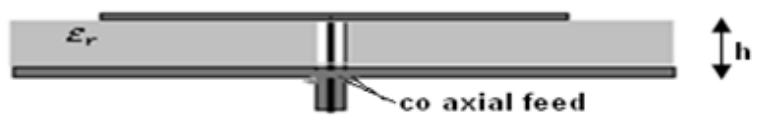

Figure.1 .C-slotted Microstrip Patch antenna

the vertical slot length, $\mathrm{W}_{2}$ and $\mathrm{W}_{3}$ are the upper and lower vertical patch length respectively. Figure1. shows the layout of a coaxial probe-fed C-slotted patch antenna.

The four dimensions $\mathrm{L} 1, \mathrm{~W}_{1}, \mathrm{~W}_{2}$ and $\mathrm{W}_{3}$ are varied from 0.1 to $3 \mathrm{~mm}$ and we have taken corresponding simulated resonance frequencies. The feed position is varied in very close steps varying around one sixth of the length (from microstrip centre) within $1.5 \mathrm{~mm}$ along the length. These sampled points are then scaled to remain within the range $[-1,1]$ and used as the training data for the network.

\section{NETWORK ARCHITECHTURE AND}

\section{TRAINING}

For the present work the Multi-Layer Perceptron Feed forward back Propagation neural network [12, 13] and Radial basis function Artificial Neural Network models are used. These networks can be used as a general function approximator.It can be approximate any function with a finite number of discontinuous, arbitrary well given sufficient neurons in the hidden layer. The model is trained with 100 sets of input/output data, which are obtained by IE3D software based on MoM. The model is trained for different values of parameters $\left(\mathrm{L} 1, \mathrm{~W}_{1}, \mathrm{~W}_{2}\right.$ and $\left.\mathrm{W}_{3}\right)$ to get a desired frequency.

3.1 Multi-Layer Perceptron Feed forward Back Propagation (MLPFFBP) Neural Network.

MLP networks are feed forward networks trained with the standard back propagation algorithms to achieve the required degree of accuracy.
They are supervised networks, and also they required a desired response to be trained. With one or two hidden layers they can approximate virtually any input output map.

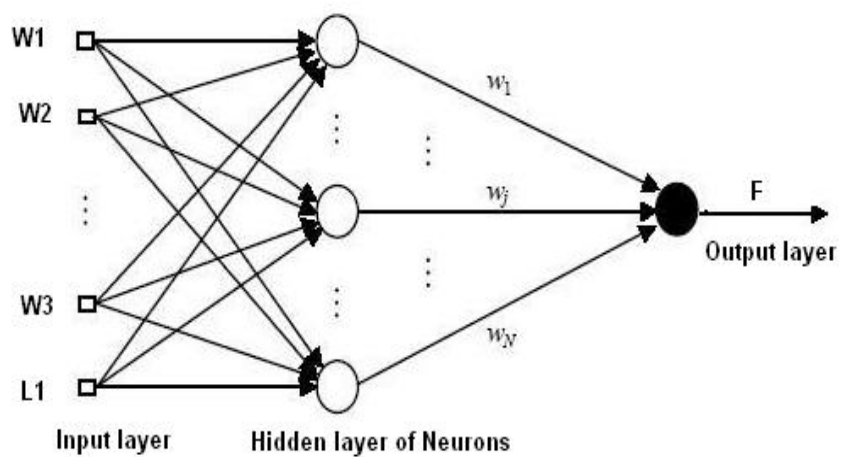

Figure 2. Three layer MLPFFBP network architecture

The weights of the networks are computed by training the network using back propagation algorithm [14, 15].In present work, the MLPFFBP (Levenberg-Marquardt) architecture shown in Figure 2.

In this network there are four input neurons in the input layer, 25 hidden neurons in the hidden layer and one output neuron in the output layer. The training time is 26 seconds and training performs in 250 epochs. In order to evaluate the performance of proposed MLPFFBP-ANN based model for the design of microstrip antenna, simulation results are obtained using IE3D simulator and generated 82 input output training patterns and 18 inputs-outputs test patterns to validate the model. During the training process the neural network automatically adjusts its weights and threshold values such that the error between predicted and sampled outputs is minimized. The adjustments are computed by the back propagation algorithm. The training algorithm most suitable is trainlm. The error goal is 0.001.The transfer function is tansig in the architecture.

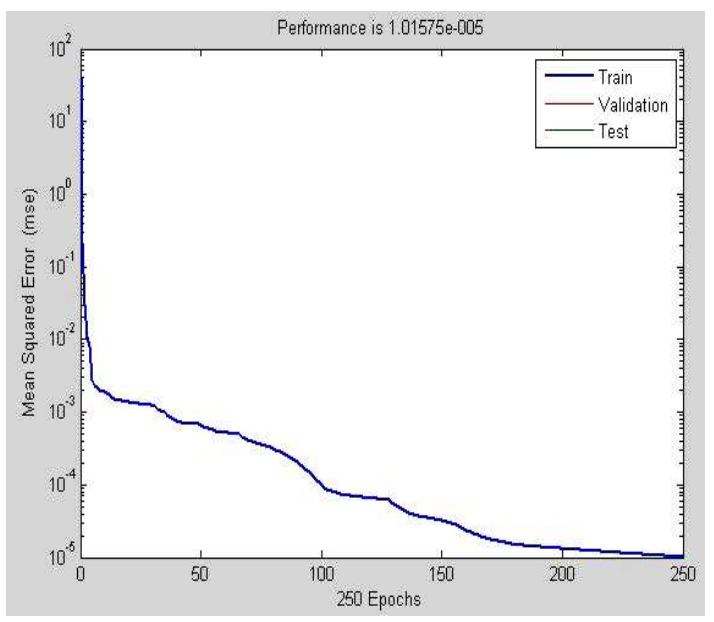

Figure 3. Number of epochs to achieve minimum mean square error level in case of MLPFFBP training algorithm 
In Table I resonant frequencies obtained using IE3D software and using MLPFFBP algorithm for different test patterns are compared and Mean square error has been calculated in Table I.

Table I: Comparison of results obtained using IE3D software and MLPFFBP -ANN algorithm

\begin{tabular}{|c|c|c|c|c|c|c|c|}
\hline $\mathbf{S}$ & $\mathbf{W}_{1}$ & $\mathbf{W}_{2}$ & $\mathbf{W}_{3}$ & $\mathbf{L l}$ & $\begin{array}{c}\mathbf{F}(\mathbf{G r}) \\
\text { IE3D }\end{array}$ & $\begin{array}{c}\text { F(GHz) } \\
\text { ANN }\end{array}$ & MSE \\
1 & 7.5 & 6.7 & 5.8 & 9.3 & 6.884 & 6.884 & 0.0001 \\
2 & 7 & 6 & 7 & 9 & 6.869 & 6.876 & 0.0585 \\
3 & 7 & 6.8 & 6.2 & 9 & 6.883 & 6.884 & 0.0012 \\
\hline 4 & 6.4 & 6.4 & 7.2 & 9 & 6.876 & 6.875 & 0 \\
5 & 7.2 & 6.2 & 6.6 & 9.2 & 6.876 & 6.878 & 0.0039 \\
6 & 7 & 6.5 & 6.5 & 9.3 & 6.884 & 6.883 & 0.0001 \\
7 & 7.1 & 6.6 & 6.3 & 9.3 & 6.884 & 6.884 & 0.0009 \\
\hline 8 & 7.2 & 6.8 & 6 & 9.3 & 6.874 & 6.873 & 0 \\
9 & 7.4 & 6.9 & 5.7 & 9.4 & 6.876 & 6.876 & 0 \\
\hline 10 & 7.1 & 7.1 & 5.8 & 9.1 & 6.884 & 6.884 & 0 \\
\hline 11 & 7.4 & 6.4 & 6.2 & 9.8 & 6.876 & 6.875 & 0.0001 \\
12 & 6.5 & 6.4 & 7.1 & 9.8 & 6.876 & 6.876 & 0 \\
\hline 13 & 6.4 & 6.5 & 7 & 9.7 & 6.876 & 6.876 & 0 \\
14 & 6.6 & 6.6 & 5.8 & 9.6 & 6.874 & 6.874 & 0 \\
\hline 15 & 6.6 & 6.6 & 5.8 & 9.4 & 6.884 & 6.883 & 0 \\
\hline 16 & 7.3 & 6.3 & 5.4 & 9.2 & 6.876 & 6.876 & 0 \\
\hline 17 & 7 & 6 & 7 & 9 & 6.884 & 6.876 & 0.0541 \\
\hline 18 & 7.2 & 6.2 & 6.6 & 9.2 & 6.876 & 6.878 & 0.0039 \\
\hline
\end{tabular}

Figure 3.shows the training performance of the developed neural model for proposed antenna using MLPFFBP algorithm. Model is trained in 250 epochs and the training time was 26 seconds.

\subsection{Radial basis function Artificial Neural Network}

Radial basis function network $[16,17]$ is a feed forward neural network with a single hidden layer that use radial basis activation functions for hidden neurons. RBF networks are applied for various microwave modeling purpose. The RBF neural network has both a supervised and unsupervised component to its learning. It consists of three layers of neurons-input, hidden and output. The hidden layer neuron represents a series of centers in the data space. Each of these centers has an activation function, typically Gaussian. The activation depends on the distance between the presented input vector and the centre. The farther the vector is from the centre, the lower is the activation and vice versa [12].The generation of the centre and their widths is done using an unsupervised k-means clustering algorithm. The centre and widths created by this algorithm then form the weights and biases of the hidden layer, which remain unchanged once the clustering has been done.
The typical RBF network structure is given in Figure 4

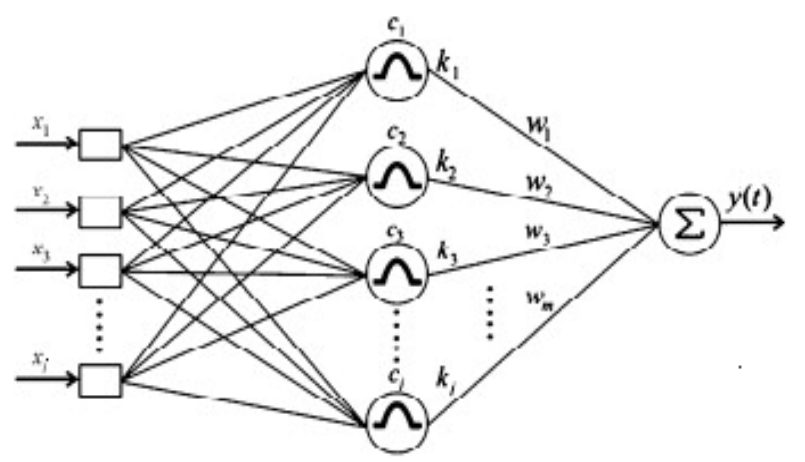

Figure 4: RBF-NN Basic Structure

The parameters ci and $\varepsilon \mathrm{i}$ are centre and standard deviations of radial basis activation functions. Commonly used radial basis activation functions are Gaussian and Multidquadratic.

Given the input $\mathrm{x}$, the total input to the ith hidden neuron $\gamma \mathrm{i}$ is given by -

$$
\gamma_{i}=\sqrt{\sum_{j=1}^{m}\left(\frac{x_{i}-c_{i}}{\gamma_{i}}\right)}, t=1,2,3, \ldots . N
$$

Where $\mathrm{N}$ is the number of hidden neurons. The output value of the ith hidden neuron is $\mathrm{Zi}=\sigma(\gamma \mathrm{i})$, where $\sigma(\gamma \mathrm{i})$ is a radial basis function. Finally, the output of the RBF network are computed from hidden neuron is given by-

$$
y_{k}=\sqrt{\sum_{i=0}^{N} w_{i} k_{i}}, k=1,2,3, \ldots . N
$$

Where wi is the weight of the link between the ith neuron of the hidden layer and kth neuron of the output layer.

In RBF network, the spread value was chosen as 0.01 , which gives the best accuracy. The network was trained with 82 samples and tested with 18 samples. In the structure there is one input and one output was used for the analysis ANN.

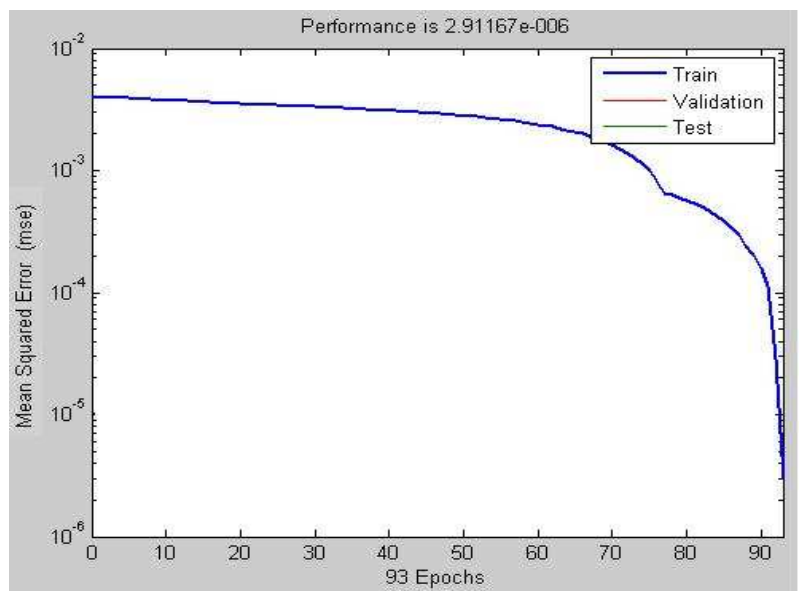

Figure 5. Number of epochs to achieve minimum mean square error level in case of RBF network. 
The RBF network automatically adjusts the number of processing elements in the hidden layer till the defined accuracy is reached. The training algorithm is unsupervised $\mathrm{k}$-means clustering algorithm.

Figure 5. shows the training performance of the developed neural model using RBF Network. Model is trained 93 epochs.

It is clear that RBF network is much faster than feed forward networks since RBF network is trained fewer epochs than feed forward network

\section{RESULTS AND DISCUSSION}

The antenna fabricated for validation of ANN are simulated using IE3D simulation software for comparison and validation purpose of ANN, because the specification of these antennas are not included in the training data base. The results of IE3D simulation software and MLPFFBP (Levenberg-Marquardt) training algorithm is shown in Figure 6.

It has been established from Figure 3.that the MLPFFBP training algorithm is the optimal model to achieve desirable values of speed of convergence. It has been observed that 250 epochs are needed to reduce MSE level to a low value $1.01575 \mathrm{e}-005$ for three layers MLPFFBP with transig as a transfer function. Achievement of such a low value of performance goal (MSE) and accuracy of $99.89 \%$ indicates that ANN model is an accurate model for designing the Microstrip patch antenna. It is observed that tansig is most suitable transfer function for present work. Minimum MSE is calculated after training the network and indicated in Table I

As the work signifies RBF-ANN is also used to model the Microstrip patch antenna. A Radial Basis Function neural network has an input layer, a hidden layer and an output layer. The neurons in the hidden layer contain Gaussian transfer function whose outputs are inversely proportional to the distance from the centre of the neuron. It is established from Figure 5. That RBF is giving results not only more accurate but fast also, the presented RBF network has performed training in less epochs than MLPFFBP.So it is concluded that RBF architecture is better from MLPFFBP to the accuracy of $99.97 \%$ and quite faster comparatively.

\section{CONCLUSIONS}

Artificial neural network structure is used for analysis of C-slotted Microstrip patch antenna characteristics. Multilayer Perceptron trained in the backpropagation mode (using Levenberg-Marquardt algorithm) and Radial basis Function Network model are developed. The important characteristics namely, resonance frequencies obtained with the present techniques are closer to the experimental results generated by simulating a large no. of C-slotted microstrip patch antenna using IE3Dsoftware.The comprehensive comparison found that RBF network is better than MLPFFBP network in prediction accuracy, training time and training speed. It proved that the RBF neural network is more efficient and accurate than MLPFFBP neural network. The developed neural network methodology can be extended for characterizing other different shapes of Microstrip antenna.

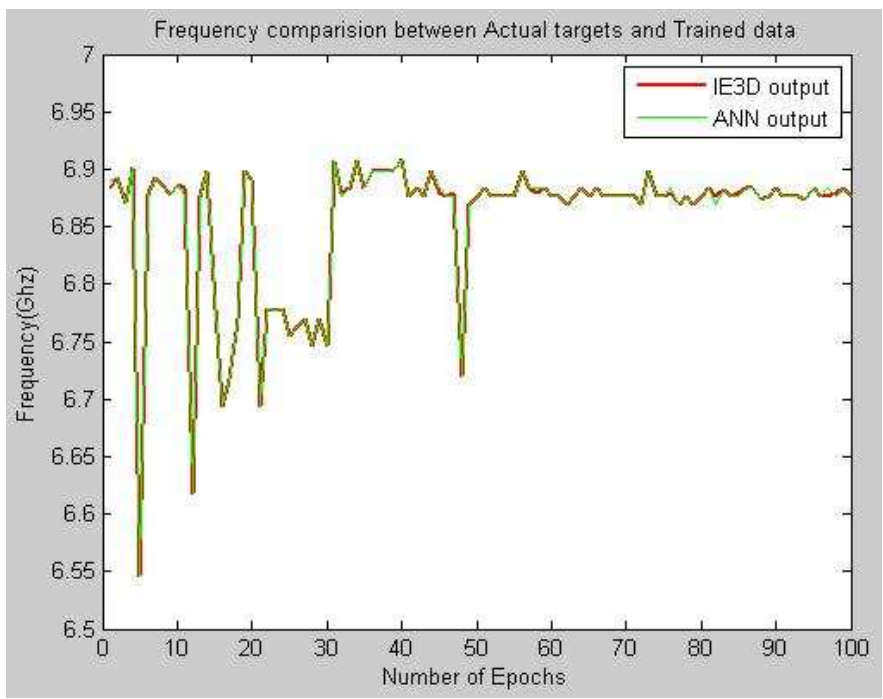

Figure 6. Comparison of frequency of antenna obtained using IE3D simulation software and Trained with MLPFFBP.

\section{REFERENCES}

[1] Balanis, C. A., Antenna Theory, John Wiley \& Sons, Inc., 1997. J. Clerk Maxwell, A Treatise on Electricity and Magnetism, 3rd ed., vol. 2. Oxford: Clarendon, 1892, pp.68-73.

[2] P. K. Singhal and L; Srivastava, "On the investigations of a wide band proximity fed bow tie shaped microstrip antenna", Journal of Microwave and Optoelectronics, Vol; 3, pp; 87-98, April 2004.

[3] Thakare V.V.,Singhal P.K.,"Bandwidth Analysis by introducing slots in microstrip antenna design using ANN " , Progress In Electromagnetic Research M., Vol. 9,107-122,2009.

[4] Qi-Jan Zhang,Senior Member,IEEE,Kuldip C.Gupta, Fellow,IEEE, and Vijay K. Devabhaktuni, Student Member,IEEE"Artificial Neural Networks for RF and Microwave Design-From Theory to Practice", IEEE Transaction on Microwave Theory and Techniques, Vol. 51, No. 4,April 2003.

[5] Patnaik,A.;Anagnostou, D.;Christodoulou ,C.G.; Lyke, J.C., "Neurocomputional Analysis of a Multiband Reconfigurable Planar Antenna", IEEE Transaction on Antennas and Propagation, Vol. 53, No. 11, November 2005,p. 3453-3458.

[6] Patnaik,A.;Anagnostou, D.;Christodoulou ,C.G., "Neural Networks in Antenna Engineering- Beyond Black -Box Modeling", IEEE/ACES International Conference on Wireless Communication and Applied Computional Electromagnetics, April 2005,p. 598-601.

[7] Patnaik,A.;Mishra, R,K., "ANN Techniques in Microwave Engineering" IEEE Microwave Magazine, Vol. 1,No. 1, March 2000,p 55-60.

[8] Vegni,L.; Toscano, A.,"Analysis of Microstrip Antennas Using Neural Networks", IEEE Transactions on Magnetics, Vol. 33,No. 2, March 1997,p. 14141419.

[9] Mishra, R,K.; Patnaik,A. “ Neurospectral Analysis of Coaxial Fed Rectangular Patch Antenna ".IEEE 
Antennas and Propagation Society International Symposium, Vol. 2, July 2000,p. 1062-1065.

[10]

Patnaik,A.;Anagnostou,D.E;Mishra,R.K.;Chistodoulou, C.G.; Lyke, J.C., "Applications of Neural Networks in Wireless Communications"IEEE Antennas and Propagation Magazine, Vol. 46,No. 3, June 2004,p. 130-137.

[11] C.A.Balanis,"Antenna Theory and Design", 2nd Edition, New York, Wiley 1997.

[12] Haykin,S., Neural Networks, 2nd edition,PHI,2003.

[13] Hassoun, M.H., Fundamentals of Artificial Neural Netorks ,Chepter 8, New Delhi,Prentice Hall of India, 1999. [14] S.Rajasekaran, G. A. Vijayalakshmi Pai, "Neural
Networks,Fuzzy Algorithms:Synthesis and Application", Prentice Hall of Limited, New Delhi ,2006.

[15] Hagan,M.T.; Menhaj, M. B., "Training Feedforward Networks with the Marquardt Algorithm". IEEE Transaction on Neural Networks, Vol. 5, No. 6, November 1994, p. 989-993.

[16] Thakare V.V.., Jadon, Shalendra Singh, Kumari, Ritesh,"Analysis of Circular Microstrip Patch Antenna Using Radial Basis Artificial Neural Network", International Journal of Electronics and Computer Science Engineering, ISSN-2277-1956

[17] Vandana Vikas Thakare, Pramod Singhal, "Artificial Intelligence in the estimation of patch dimensions of Rectangular Microstrip Antennas" .Circuits and Systems, Oct. 2011, Vpol. 2, pp. 330-337.

\section{BIOGRAPHIES}

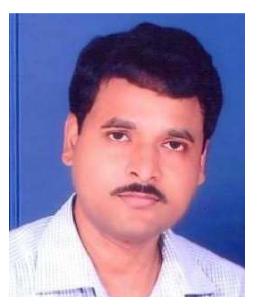

Mr. Pritam Singha Roy ,Assistant Professor in DIET and got M.Tech degree in Electronics and Control Engineering,Calcutta University,India, in 2009. His current areas of interest are design and development of microstrip ,dielectric and metallic antennas.

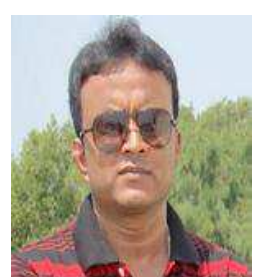

Dr. samik Chakraborty ,Professor at Indian Maritime University,India..He received $\mathrm{Ph} . \mathrm{D}$ (Science) in 2009 ,from Jadavpur University,Kolkata ,India.He is currently supervising two doctoral thesis.He has worked in different research projects for National Defence and Space Research Organizations 